\title{
L'ecografia renale nello studio del paziente con insufficienza renale cronica
}

\author{
M. Zanazzi, R. Scarpioni \\ Seconda Divisione Medica - Ospedale Civile di Piacenza
}

L'ecotomografia ha ampiamente migliorato la diagnostica del paziente con insufficienza renale (1). Utilizzata come indagine di prima istanza nello studio delle nefropatie per l'elevata affidabilità diagnostica, l'ecografia renale fornisce un'informazione immediata sulla presenza, grandezza, forma e sede dei reni. Questa metodica permette di differenziare le nefropatie parenchimali da quelle secondarie a nefropatia ostruttiva, e può essere utilizzata in tutti i pazienti nefropatici, indipendentemente dal grado di insufficienza renale. L'ecotomografia renale rappresenta pertanto un eccellente metodo per:

a) valutare l'entità del danno renale

b) documentare l'evoluzione della nefropatia.

I parametri di valutazione ecografica renale abitualmente indagati in corso di insufficienza renale cronica sono:

1) DiMENSIONI

2) MorfologiA

3) SimmetriA

4) ECOGENICITÀ PARENCHIMALE

5) SPessore CORTICALE

6) DIFFERENZIAZIONE CORTICO-MIDOLLARE

\section{Insufficienza renale cronica parenchimale}

Da sempre nel campo delle nefropatie mediche, lo studio ultrasonografico è stato finalizzato al tentativo di formulare una diagnosi circostanziata. Nelle forme di insufficienza renale cronica parenchimale avanzata frequente è il riscontro di un rene atrofico (2), con diametro bipolare ridotto, contorni finemente irregolari o lobulati dalla presenza di aree transoni- che a sviluppo esorenale, ridotto spessore corticale, perdita della differenziazione corticomidollare, iperecogenicità diffusa o distrettuale della corticale, accentuazione dell'ecogenicità pielica (Fig. 1). Tale quadro è aspecifico e solo raramente è possibile proporre una plausibile ipotesi diagnostica. Sebbene sia sempre necessaria una conferma istopatologica per una diagnosi delle nefropatie mediche, l'ecotomografia renale permette di caratterizzare un'ampia gamma di quadri clinici.
Fig. 1 - Rene destro con i caratteri ecotomografici del rene atrofico. 
Le dimensioni renali possono risultare ridotte, in corso di:

\section{GLOMERULONEFRITI CRONICHE}

NEFROPATIE INSTERSTIZIALI

NEFROANGIOSCLEROSI

NEFRONOFTISI

NEFROCALCINOSI

oppure normali o aumentate, in corso di:

RENE POLICISTICO

AMILOIDOSI

Mieloma

Nefropatia diabeTiCA

(nelle fasi iniziali)

\section{LOCALIZZAZIONI LINFOMATOSE RENALI (bilaterali)}

\section{GLOMERULONEFRITE MEMBRANOSA.}

Tra le nefropatie parenchimali con reni di dimensioni aumentate ricordiamo il rene policistico dell'adulto e tra quelle di ridotte dimensioni la malattia cistica acquisita dell'uremico e del dializzato.

\section{Rene policistico dell'adulto}

Tale patologia (3) è più propriamente definita malattia policistica (a trasmissione autosomica dominante) per la frequente associazione di lesioni cistiche renali, ma anche epatiche e più raramente spleniche ed ovariche $(4,5)$. Il quadro ecografico (Fig. 2) tipico è di reni di dimensioni notevolmente aumentate, con diametro bipolare superiore a $15 \mathrm{~cm}$ (sino a 3-4 volte le normali dimensioni), profili bozzuti per la presenza bilateralmente di multiple formazioni cistiche, compresse le une contro le altre. Il volume delle formazioni cistiche non è solitamente superiore a $5 \mathrm{~cm}$ di diametro, risultando ubiquitarie, con evidenza del seno renale conservato solo nelle fasi iniziali della malattia. L'incidenza della malattia policistica dell'adulto è di 1:1000 soggetti, con la comparsa di insufficienza renale generalmente nella $4^{\text {a }} 5^{\text {a }}$ decade di vita. È pertanto necessario uno screening ecografico familiare per l'individuazione precoce della patologia, ancor prima del manifestarsi dei sintomi. Nel follow-up del rene policistico è necessario individuare la presenza di formazioni cistiche complicate da infezioni o emorragie (4). La presenza di echi di bassa e media intensità con tendenza alla sedimentazione, orientano più frequentemente verso un processo flogistico. Echi più grossolani ad elevata intensità, con disposizione disomogenea, possono porre problematiche di diagnostica differenziale tra un processo emorragico ed un processo neoplastico (6).

\section{Malattia cistica acquisita dell'uremico e del dializzato}

La malattia renale cistica acquisita (Fig. 3 ), dapprima riscontrata in pazienti in cronico trattamento dialitico (7), è stata successivamente segnalata in pazienti in CAPD (8), negli uremici cronici (9) ed in quelli sottoposti a trapianto (10).

Patologia dapprima considerata come complicanza diretta del trattamento extracorporeo, attualmente viene considerata la normale evoluzione delle nefropatie croniche a diversa eziologia. La sua frequenza è stata riportata in percentuali variabili dal 10 al $95 \%$ nelle varie casistiche (5-8). La patogenesi della malattia risulta sconosciuta, anche se sono state indicate diverse possibili cause nella genesi delle formazioni cistiche (ad esempio sostanze tossiche legate all'uremia ed alla dialisi (9-11), processi ischemici reiterati a livello renale (9), ostruzione tubulare (9-12)). La malattia cistica ac-

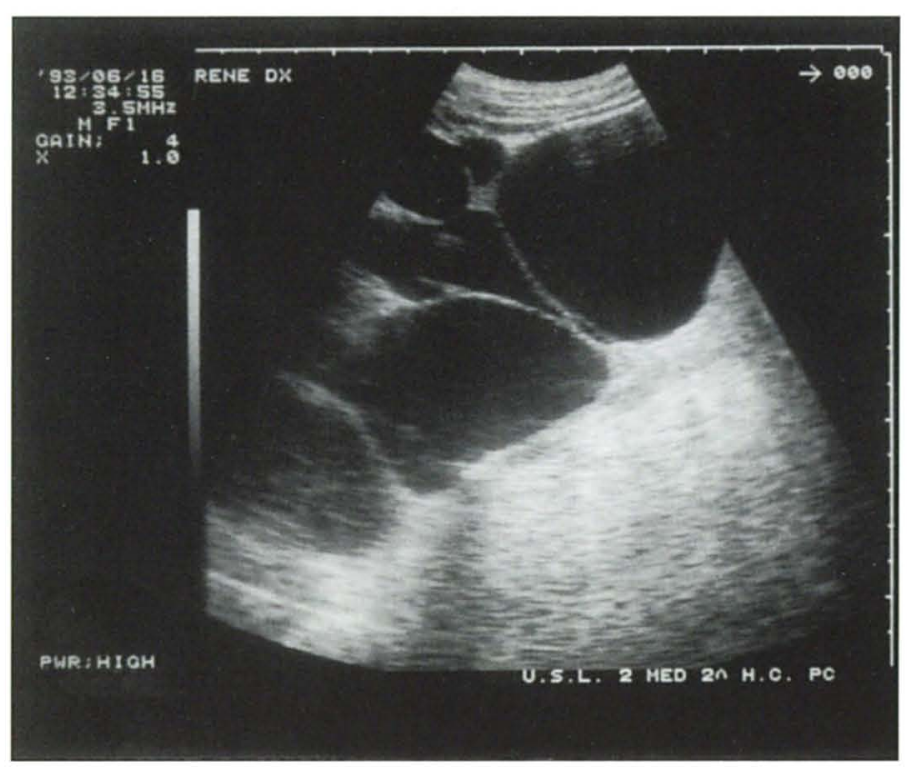

Fig. 2 - Rene destro. Rene policistico dell' adulto.

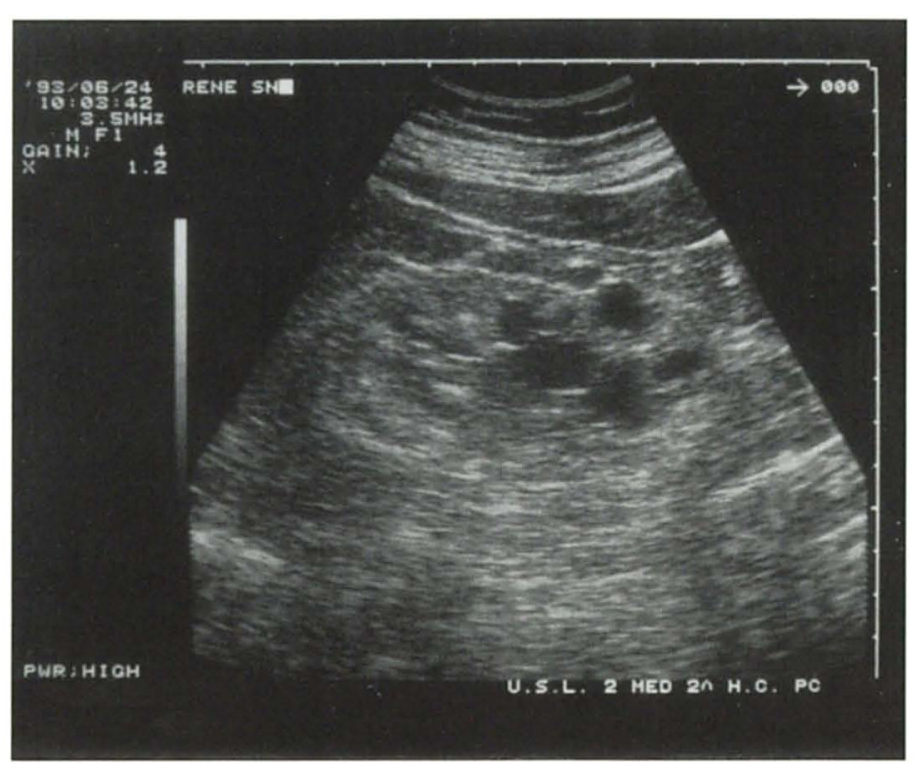

Fig. 3 - Rene sinistro. Malattia cistica acquisita del dializzato. 
quisita renale può essere considerata condizione precancerosa $(13,14)$ per il significativo rischio di degenerazione neoplastica (con incidenza 2500 volte superiore a quella dei pazienti con normale funzione renale).

L'incidenza di tale complicanza nei pazienti con insufficienza renale cronica terminale, ma soprattutto nei pazienti emodializzati o sottoposti a dialisi peritoneale od a trapianto renale, consiglia un'indagine ecotomografica di screening (affiancato eventualmente alla TC) ed un successivo monitoraggio ecografico semestrale. Particolare attenzione deve essere posta in presenza di:

macroematuria

trattamento emodialitico superiore a 3 anni

masse solide di difficile interpretazione (oltre all'indagine ecotomografica ed alla TC indicata l'esecuzione di citoaspirato ecoguidato) (15).

Anche i pazienti sottoposti a trapianto renale (16) devono essere valutati attraverso periodiche indagini ultrasonografiche perché nonostante la normalizzazione della funzione renale, l'evoluzione cistica può proseguire nel rene nativo, quando non asportato.

Tale trasformazione sembrerebbe strettamente legata alla durata della dialisi prima del trapianto. Nella malattia cistica acquisita renale il quadro ecografico è caratterizzato da reni di ridotte dimensio- ni (solo raramente di aumentate dimensioni, in corso di complicanze o degenerazioni neoplastiche), con aree anecogene multiple, mono o bilaterali, distribuite casualmente nel parenchima renale, a prevalente sede corticale.

\section{Morfologia}

All'ecotomografia i reni, in corso di insufficienza renale cronica possono presentarsi a contorni regolari nelle:

\section{GLOMERULONEFRITI CRONICHE}

\section{NEFROANGIOSCLEROSI}

\section{NEFROPATIE INTERSTIZIALI DA FARMACI}

oppure a contorni irregolari nelle:

\section{PIELONEFRITI CRONICHE}

\section{NeFropatiE DA REFLUSSO}

\section{RENE POLICISTICO NELL'ADULTO}

Tratteremo due patologie renali con differenti aspetti morfologici, l'una con reni a contorni irregolari (pielonefriti croniche) e l'altra con reni a contorni regolari (pielonefrite interstiziale da farmaci).

\section{Pielonefriti croniche}

Le pielonefriti croniche sono frequenti in età pediatrica (nefropatia da reflusso) (Figg. 4, 5) ed hanno l'incidenza massima durante il periodo fertile della donna (17) (importanza della gravidanza come fattore favorente) e nella senilità dell'uomo (legate all'ipertrofia prostatica). L'ecografia dimostra oltre ad una riduzione di volume, reni alterati nella forma, con avvallamenti e protuberanze corrispondenti rispettivamente ad aree di atrofia cicatriziale e di ipertrofia parenchimale. L'esistenza di un'area focale di danno parenchimale viene pertanto evidenziata come una percettibile riduzione del parenchima renale, riferibile da un punto di vista anatomopatologico, ad area atrofica e fibrotica. Nella pielonefrite cronica più frequentemente tali retrazioni sono rilevate bilateralmente (tipico ne è il riscontro nei pazienti diabetici di mezza età nei quali è possibile evidenziare anche lesioni papillari). Esistono una serie di situazioni che possono condurre ad un'errata diagnosi di raggrinzimento ed atrofia (18).

E ben nota la differenziazione tra le incisure dovute a persistenza delle lobature fetali e quelle dovute a raggrinzimenti; nel primo caso le incisure del profilo esterno del rene si verificano tra i calici. Nel soggetto adulto sano può essere vi-

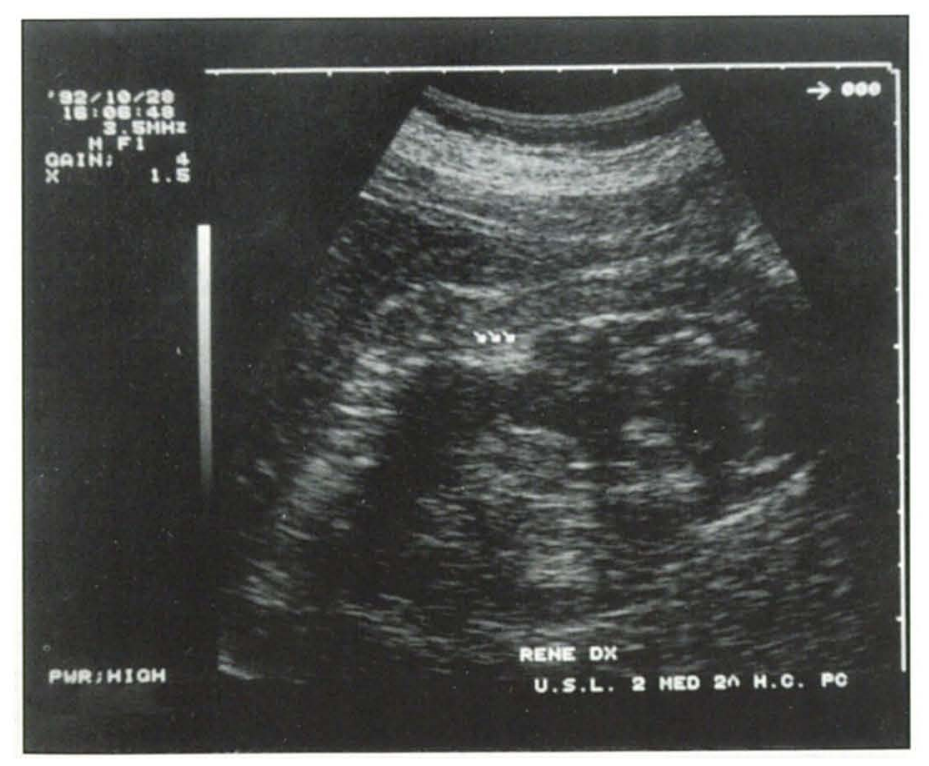

Fig. 4 - Rene destro. Nefropatia da reflusso.

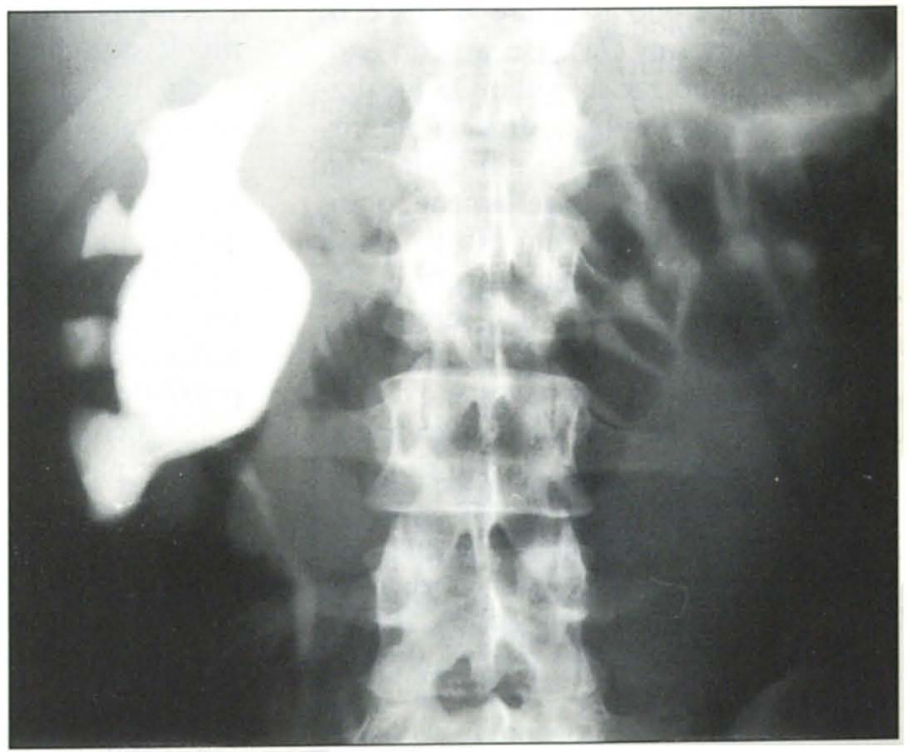

Fig. 5 - Nefropatia da reflusso, urografia. 


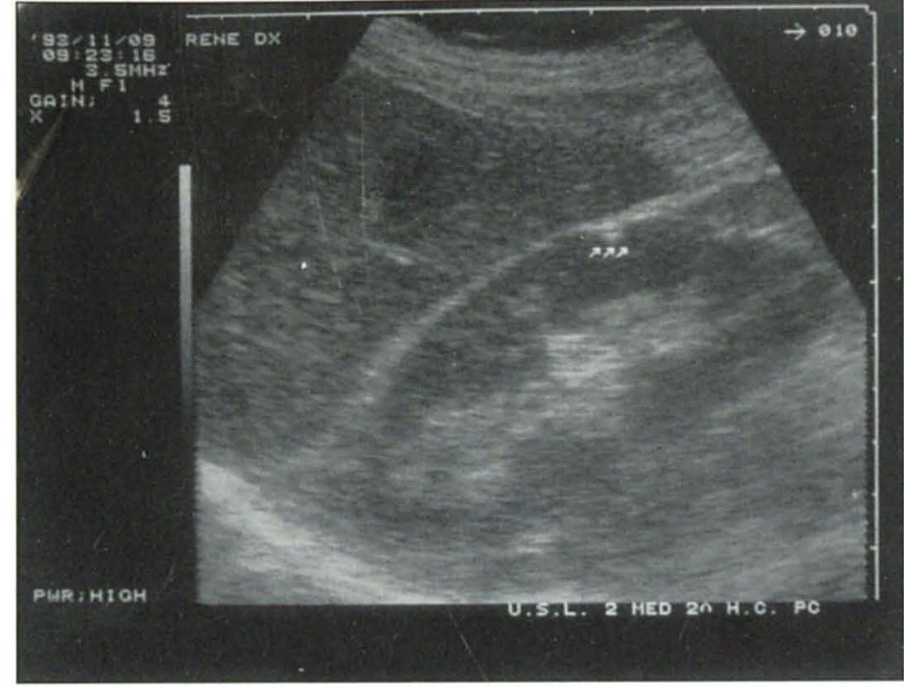

Fig. 6 - Rene destro. Triangolo iperecogeno, soggetto normale.

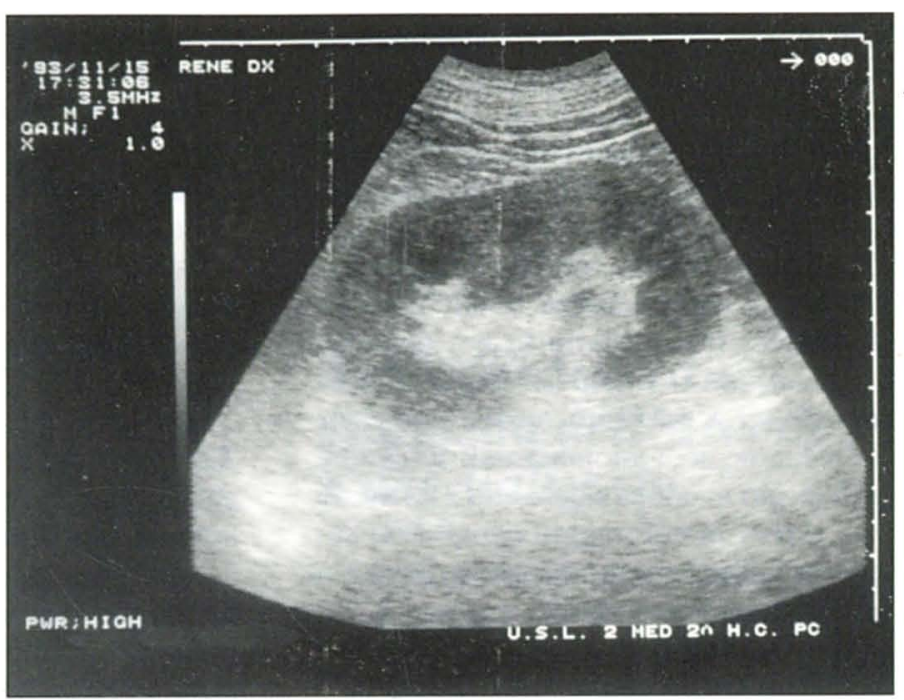

Fig. 7 - Rene destro. Insufficienza renale iniziale in corso di amiloidosi. sualizzato il triangolo iperecogeno (Fig. 6), elemento anatomico ben differenziabile da una retrazione cicatriziale. Si tratta di un'area triangolare iperecogena (19) situata lungo il profilo renale più frequentemente a livello antero-superiore, più raramente a livello postero-inferiore. In corso di pielonefrite cronica, oltre al rilievo di contorni grossolanamente irregolari, è frequente il riscontro di calicectasia di vario grado (20). Tra le forme flogistiche ad andamento cronico ricorrente la più conosciuta è la tubercolosi renale.

Tale infezione può assumere aspetti ecografici multiformi (21). Un aspetto ecografico che può far sospettare la localizzazione tubercolare è la dilatazione circoscritta di un distretto caliciale, con struttura interna non perfettamente omogenea, ma contenente echi diffusi. Altre forme che possono essere ascritte alla TBC sono rappresentate dall'idronefrosi tubercolare, indistinguibili da una idronefrosi aspecifica, dalle caverne e dal rene mastice. Le caverne tubercolari hanno l'aspetto di lesioni parenchimali simil-cistiche; il contenuto può essere perfettamente ecoprivo come quello di una cisti semplice, oppure variamente ecogeno tale da simulare formazioni solide. Il rene mastice è di dimensioni ridotte, trasformato in sacche, con a livello del seno pielico, zone di iperecogenicità secondarie alla fibrosi. L'ecografia ha un ruolo di primo piano nello studio delle pielo- nefriti (aspecifiche e specifiche), sia nel cogliere la presenza di lesioni parenchimali, sia la presenza di associata dilatazione delle vie escretrici. Risulta pertanto utile nel determinare l'entità del danno anatomico e nel follow-up delle infezioni renali.

\section{Nefropatie interstiziali da farmaci}

L'ecotomografia si è dimostrata notevolmente utile nella diagnosi di nefropatie da analgesici. Il quadro ecografico più significativo è rappresentato da calcificazioni papillari che circondano il seno renale con un caratteristico pattern completo o incompleto a ghirlanda (22). Le calcificazioni, evidenziabili bilateralmente, sono multiple e localizzate centralmente al seno renale. In alcuni casi è possibile il riscontro di idronefrosi come conseguenza dell'ostruzione ureterale da necrosi papillare. Solo poche calcificazioni dimostrano cono d'ombra posteriore. Nelle fasi iniziali con modesto quadro di insufficienza renale $\mathrm{i}$ reni appaiono di normali dimensioni, con profili regolari, modesta riduzione dello spessore corticale. Nei casi di severa insufficienza renale compare una riduzione del diametro bipolare renale, ridotto spessore corticale, aumentata ecogenicità della corticale.

\section{Ecogenicità parenchimale}

La corteccia renale presenta normalmente un'ecogenicità inferiore a quella epatosplenica e la midollare un'ecogenicità inferiore a quella della corteccia renale (2). In realtà nel corso della vita il rene modifica le proprie caratteristiche acustiche. I reperti più rappresentativi del rene nell'anziano sono lo sviluppo di tessuto sclerotico con riduzione della struttura parenchimale e una accentuazione dell'ecogenicità del seno pielico (23). La corteccia è meno ecogena o isoecogena rispetto a fegato e milza e spesso non è distinguibile la midollare, che ha perduto l'anecogenicità per aumentata fibrosi interstiziale. Anche se la caratterizzazione del parametro ecogenicità parenchimale può risultare estremamente soggettivo, l'aumentata diffusa ecogenicità parenchimale può essere decisamente considerata un segno reale di nefropatia parenchimale associata a ridotta funzionalità renale. In tali occasioni solo raramente possono essere evidenziate piccole piramidi ipoecogene facilmente differenziabili dalla circostante corteccia iperecogena. Con l'aumento dell'ecogenicità della corticale anche i vasi arciformi (che determinano la definizione della giunzione corticomidollare) solo difficilmente possono essere evidenziati. Mentre non sem- 
bra esistere una correlazione diretta tra il reperto bioptico ed ultrasonografico riguardo la severità e la natura della lesione glomerulare, le alterazioni interstiziali sembrano invece essere strettamente legate all'intensità dell'ecogenicità corticale (2-6). Come già accennato, solo raramente è possibile evidenziare con l'ecotomografia la causa di una nefropatia cronica; l'indagine è più specifica nella valutazione degli stati evolutivi della malattia con modificazioni progressive dell'ecogenicità correlata con la riduzione della massa nefronica e del filtrato glomerulare. In corso di amiloidosi (Fig. 7) l'ecografia dimostra reni di dimensioni aumentate con iperecogenicità corticale diffusa, probabilmente legata alla progressiva deposizione di sostanza amiloide nei glomeruli e nell'interstizio (24).

\section{L'ecodoppler nella diagnosi delle nefropatie parenchimali}

L'ecotomografia spesso non risulta dirimente nella diagnosi delle nefropatie croniche. In questi ultimi anni è stato invece sottolineato il ruolo del doppler pulsato nella diagnosi differenziale delle nefropatie mediche. In esse si osserva spesso un aumento delle resistenze periferiche; si evidenzia cioè una riduzione più o meno marcata della componente diastolica del flusso, segno della scarsità del letto arteriolare o delle difficoltà che incontra il sangue a fluire nelle diramazioni arteriose periferiche. L'entità delle resistenze arteriolari periferiche viene convenzionalmente espressa come il rapporto tra la velocità di picco sistolico $(\mathrm{S})$ e quella telediastolica (D). (S/D) (25).

Più recentemente per definire l'entità delle resistenze periferiche è stato impiegato l'indice di resistenza (RI), che è dato dal rapporto $\mathrm{S}-\mathrm{D} / \mathrm{S}$, dove $\mathrm{S}$ è la velocità di picco sistolico e D quella telediastolica. Esisterebbe una correlazione, anche se non costante, tra RI ed il grado di insufficienza renale; è possibile comunque riscontrare tracciati velocimetrici (Fig. 8) pressoché normali in pazienti con insufficienza renale cronica avanzata (26). Alcuni autori hanno proposto, sulla base delle modificazioni dell'indice di resistenza RI, una possibile differenziazione,

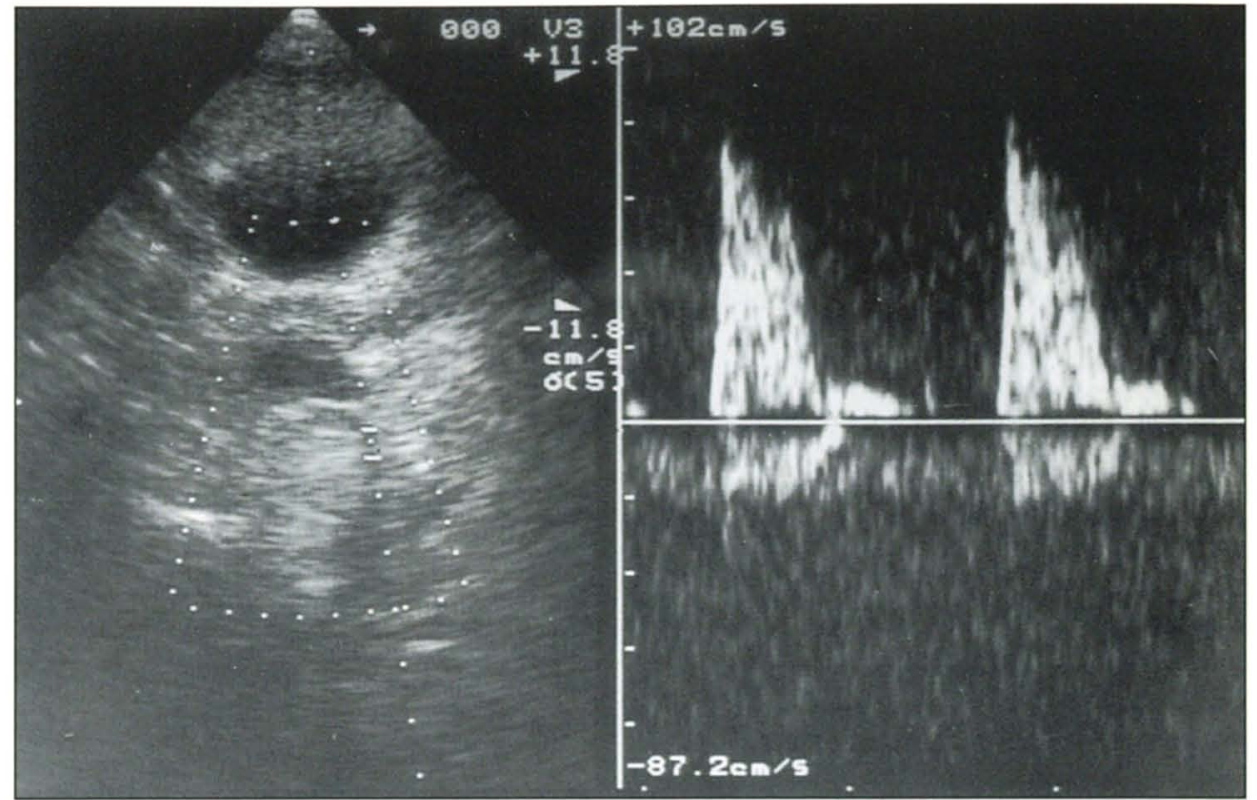

Fig. 8 - Tracciato flussimetrico di paziente monorene con stenosi di arteria renale sinistra ostiale

con buona approssimazione, tra le vasculiti e le nefropatie tubulo interstiziali da una parte e le glomerulonefriti dall'altra (27).

Nel primo gruppo si osserverebbe un aumento dell'indice di resistenza RI, in genere superiore a 0.70 ; nel secondo gruppo l'indice rimarrebbe sostanzialmente inalterato. L'interpretazione del RI deve tener conto di una certa variazione "fisiologica" nei pazienti anziani che presentano insufficienza renale cronica. Nei pazienti diabetici con insufficienza renale RI può risultare non essere legata all'insulinodipendenza, ma alla presenza di microalbuminuria, alla durata della malattia, ad un compenso glicometabolico inadeguato. Anche se attualmente sembra difficile proporre una correlazione tra RI e le lesioni istologiche osservate nella nefropatia diabetica, questo indice può divenire un elemento supplementare nel follow-up, nella ricerca e nella prevenzione della microangiopatia. L'ecodoppler rappresenta un'indagine diagnostica affidabile, non invasiva e ben tollerata nello studio del paziente con insufficienza renale su base ateromatosa (29). I criteri di selezione dei pazienti da sottoporre a valutazione ecodoppler per la ricerca di stenosi delle arterie renali da aterosclerosi, o dell'arteria renale in rene funzionante unico sono: 1) Età superiore a 50 anni; 2) Backgroung aterosclerotico con ipertensione arteriosa di vecchia da- ta; 3) Insufficienza renale senza causa evidente o aggravata dalla somministrazione di un inibitore dell'enzima di conversione. La valutazione ecodoppler deve essere condotta sulla parte ostiale e prossimale dell'arteria renale. I criteri diagnostici di stenosi emodinamicamente significativa (cioè superiore al $50 \%$ ) sono (Fig. 12): 1) Velocità di picco sistolico superiore a $100 \mathrm{~cm} / \mathrm{secondo}$; 2) Presenza di turbolenze distalmente; 3) Riduzione della componente diastolica del flusso nella porzione del vaso a monte della stenosi. L'ecodoppler non risulterebbe diagnostico nei reni con diametro bipolare inferiore a $8 \mathrm{~cm}$ e con insufficienza renale di elevato grado in quanto l'arteria renale ha solitamente calibro ridotto e quindi difficilmente valutabile. L'ecodoppler risulta ampiamente utilizzato nel follow-up di pazienti sottoposti ad angioplastica percutanea delle arterie renali. Può direttamente confermare l'avvenuta dilatazione dimostrando il recupero della curva velocimetrica, anche se possono permanere turbolenze marcate e flusso accelerato secondario a flaps intimali e ad ematomi intramurali. Nel monitoraggio è fondamentale misurare la velocità massima del picco sistolico, evidenziando nei casi a favorevole evoluzione, una progressiva riduzione della velocità del flusso con stabilizzazione dopo pochi giorni dalla dilatazione. L'innalzamento del picco massimo di 


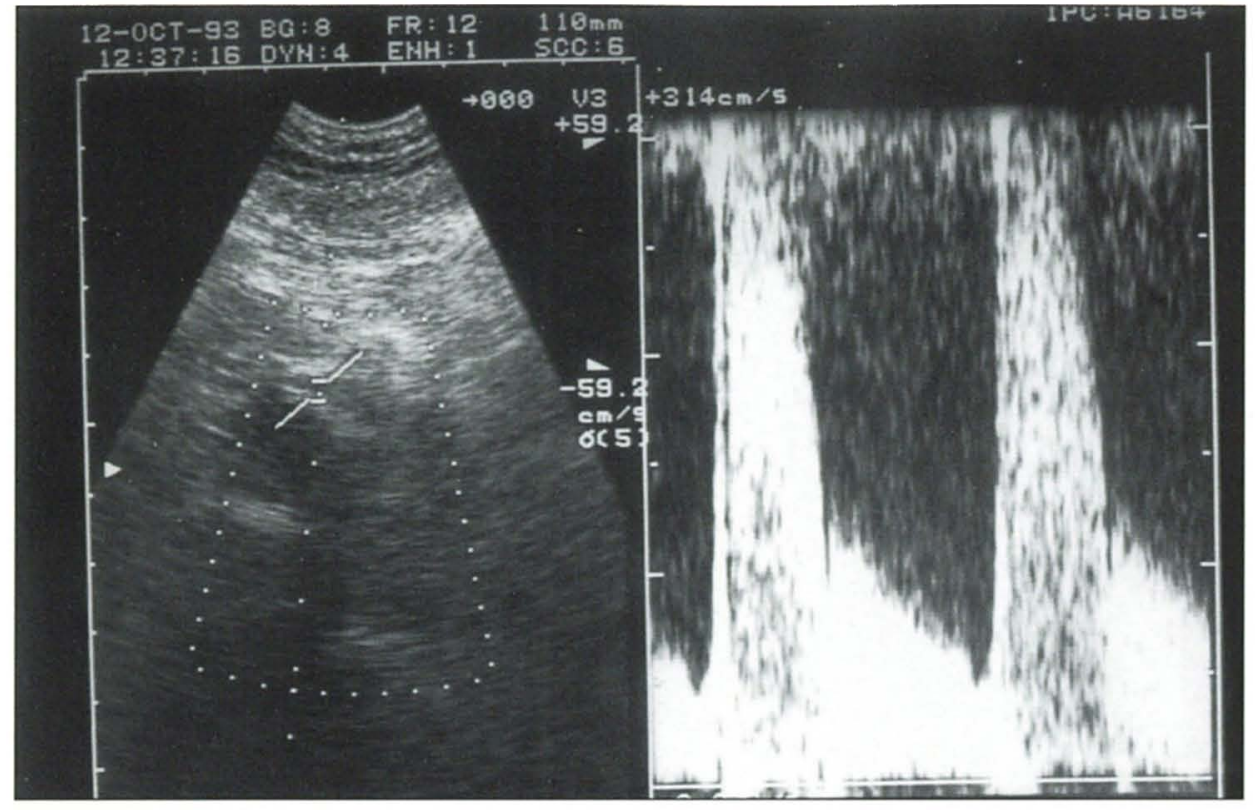

Fig. 9 - Tracciato flussimetrico di arteria renale destra. Paziente con insufficienza renale cronica parenchimale.

velocità, con incremento delle turbolenze, fa sospettare una recidiva della stenosi. Dopo angioplastica transluminale si può assistere ad embolizzazione (30) di frammenti della placca ateromasica nel distretto arterioso renale: questa embolizzazione determinerebbe l'obliterazione del letto arteriolare periferico con peggioramento dello stato ipertensivo ed evoluzione verso una progressiva insufficienza renale, sino alla fase di ERSD (end stage renal disease).

\section{Insufficienza renale cronica ostruttiva}

Per idronefrosi s'intende la dilatazione delle vie urinarie escretrici, secondaria ad ostruzione di tipo meccanico o funzionale.

Tra le cause ostruttive intrinseche ricordiamo: calcoli, tumori, stenosi congenite ed acquisite. Tra le cause ostruttive estrinseche: neoplasie, fibrosi retroperitoneale, vasi anomali, briglie aderenziali, traumi, iatrogeni.

Le cause non ostruttive sono rappresentate: 1) malformative: megacalicosi, diverticoli caliciali, megauretere; 2) acquisite: dilatazioni post ostruttive, necrosi papillare, tubercolosi, diabete insipido, reflus- si vescico-ureterali. Vengono descritti tre gradi di dilatazione della via escretrice intrarenale in base all'aspetto della stessa e allo spessore del parenchima:

1) Dilatazione lieve: nella zona centrale del seno si apprezzano piccole aree ecoprive ramificate, convergenti verso l'ampolla. 2) Dilatazione media: le aree ecoprive sono decisamente più ampie e più chiaramente visibile la connessione tra calici ed ampolla. 3) Dilatazione severa: il seno renale appare sostituito da sacche transoniche; evidente risulta la riduzione dello spessore parenchimale con aumento dell'ecogenicità (Fig. 10). Le nefropatie croniche ostruttive sono più frequentemente $(65 \%)$ di origine neoplastica (neoplasia degli organi pelvici), mentre solo in un terzo dei casi sono imputabili a cause non neoplastiche (calcolosi, fibrosi retroperitoneale, vescica neurologica). L'indicazione dell'ecotomografia in questi casi è duplice:

- come tecnica di monitoraggio,

- come tecnica di guida alla pielostomia percutanea bilaterale con detensione delle vie escretrici e, nei casi complicati da pionefrosi, la rimozione dello stato settico con l'eventuale recupero della residua funzione renale. Nelle ostruzioni complete croniche alla lenta e progressiva riduzione della filtrazione glomerulare si aggiunge un'alterazione dei processi di scambio tubulare. La protratta ipertensione endocanalicolare si ripercuote sulle regioni papillari, sui vasi adiacenti (soprattutto i venosi), determinando un aumento della pressione idrostatica dei capillari peritubulari ed una riduzione della perfusione renale per fattori ischemici. In questa situazione l'atrofia parenchimale è il fenomeno dominante, secondario a processi ischemici. L'atrofia provoca importante dilatazione della via urinaria intraparenchimale con corticalizzazione dei calici.

La diagnosi ecografica risulta pertanto agevole e questo quadro configura il terzo grado di idronefrosi.

Si desidera ringraziare il sig. Gualtiero Zucconi per la preziosa collaborazione tecnica.
Fig. 10 - Insufficienza renale cronica ostruttiva. Dilatazione severa con riduzione dello spessore parenchimale.

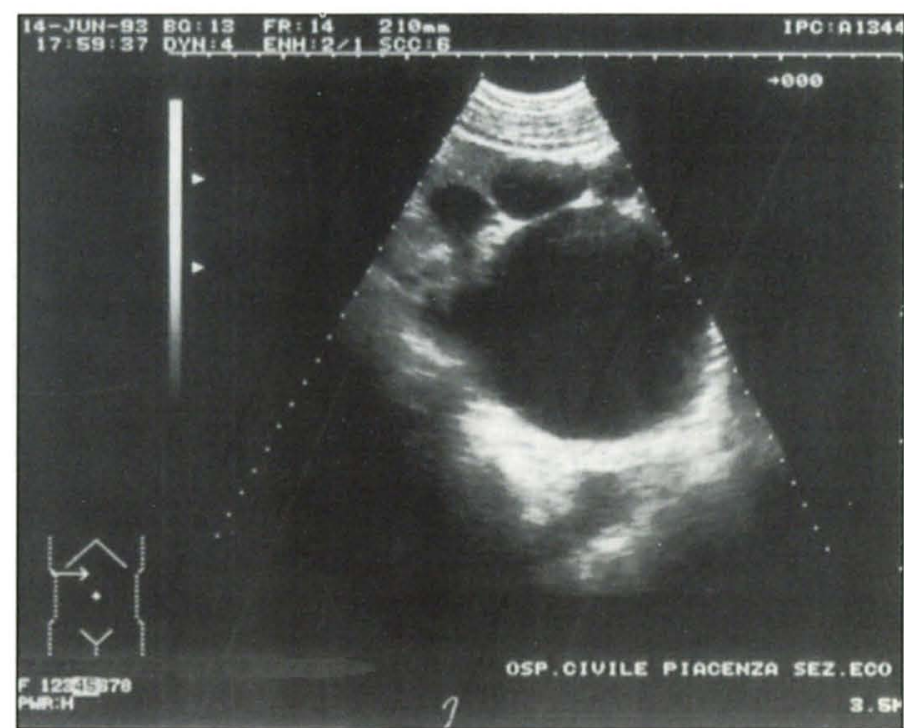




\section{BIBLIOGRAFIA}

1. Moreau JF, Ramella G, et al. Place de l'echographie ultrasonore dans l'étude des insuffisances renales de l'adulte. Annales de Radiologie 1984; 7: 27.

2. Rosenfield AT, Siegel NJ, et al. Renal parenchimal disease: Histopathologic-sonographic correlation. AJR 1981; 793: 137.

3. Parfrejs P, Bear JC, et al. The diagnosis and prognosis of autosomal dominant policystic kidney disease. N Engl J Med 1990; 1085: 323.

4. Weill FS, Bihr E, et al. L'ultrasonographie renale. Vigot 1985; 79.

5. Welling LW, Grantham JJ, et al. Cystic and developmental disease of the kidney. Brenner-Rector 1991; vol. 2: 1657

6. Hricack H, et al. Geniturinary ultrasound. Churchill Livingstone 1986.

7. Taylor AJ, Cohen EP, et al. Renal imaging in long term dialysis patients: a comparaison of CT and sonography. AJR 1989; 765: 153.

8. Trabucco A, Johansson SL, et al. Neoplasia and acquired renal cystic disease in patients undergoing CAPD. Urology 1990; 1: 25.

9. Cohen EP, Elliot WC. The role of ischemia in acquired cystic kidney disease. Am J Kidney Dis 1990; 55: 1 .

10. Ishikawa I, Yuri T, et al. Regression of acquired cystic disease of the kidney after successful renal transplantation. A M J Nephrol 1983; 310: 3 .

11. Crocker JFS, Mc Donald ATJ, et al. Haemodialysis induced policistic kidney disease: an animal model. Pediatric Res 1983; 347: 17.

12. Krempien B, Ritz E. Acquired cystic transformation of the kidneys of haemodialyzed patients. Wirchows Arch 1980; 189:386.

13. Ishikawa I, Saito Y, et al. Development of acquired cystic disease and adenocarcinoma of the kidney in glomerulonefritic chronic haemodialysis patients. $\mathrm{Cl}$ Nephrol 1980; 14: 1 .

14. Mikisch O, Bommer J, et al. Multicystic transformation of kidney in chronic renal failure. Nephron 1984; 93: 38 .

15. Bretan P, Busch M, et al. Chronic renal failure: a significant risk factor in the development of acquired renal cysts and renal cell carcinoma. Cancer 1986; 1871: 57.

16. Varizi ND, Darwish DC, et al. Acquired renal cystic disease in renal transplant recipients. Nephron 1984; $203: 37$.

17. Harber MJ, Asscher AW. Virulence of urinary pathogens. Kidney Int 1985; 717: 28.

18. Saxton HM. Processi cicatriziali del rene: cause e meccanismi . In Pistolesi GF: La radiologia del rene. Verona. Libreria Cortina 1981; 244.

19. Carter AR, Morgan JG, et al. Junctional parenchimal defect: sonographic variant of renal anatomy. Radiology 1985; 144: 449.

20. Gedroyc WMW, Chaudhuri R, et al. Normal and near normal caliceal patterns in reflux nephropathy. Clin Radiol 1988; 615: 39.

21. Piccirillo M, Rigsby CM, et al. Sonography of renal inflammatory disease. Urol Radiol 1987; 66: 9.

22. Segasothy M, Suleiman AB, et al. Paracetamol: a cause for analgesic nephropathy and end stage renal disease. Nephron 1988; 50: 50.

23. Spital A, Valvo JR, et al. Nondilated obstructive uropathy. Urology 1988; 28: 140.

24. Subramanyam BR. Renal amyloidosis in juvenile rheumatoid arth- ritis: sonographic features. AJR 1981; 136: 411.

25. Ferretti G, Salomone A, et al. Ecodoppler: metodica diagnostica non invasiva nello studio del distretto nefrovascolare. Ultrasuonodiagnostica UI 1985; 49.

26. Dubbin PA. Doppler ultrasound in renal disease. Curr Imaging 1989; 1: 32.

27. Platt JF, Ellis JH, et al. Intrarenal arterial doppler sonography in patients with non obstructive renal disease: correlation of resistive index with biopsy findings. AJR 1990; 151: 317.

28. Sauvain JL, Bourscheid D, et al. Echo-doppler pulsé des artères intra-renales. Annales de radiologie 1991; 34: 4.

29. Buchet P, Minvielle F, et al. Interet diagnostique du doppler pulsé dans l'insuffisance renale atheromateuse. Arch Mal Coeur 1991; 1191: 84 .

30. Harrington JT, Sommers SC, et al. Atheromatous emboli with progressive renal failure. Renal arteriography as the probable inciting factor. Ann Intern Med 1968; 68: 152. 